\title{
A CORRECTION TO KING'S MODEL OF STAR CLUSTERS
}

\author{
G. A. Saiyan
}

In the framework of the modified isothermal model of King, a value is obtained for the quantity $A$ which occurs in the estimate of the central density of spherical star clusters $\left(\rho_{0}=A \sigma^{2} / 4 \pi G r_{c}^{2}\right.$, where $\sigma^{2}$ is the one-dimensional velocity dispersion of the stars, and $r_{c}$ is the radius of the core). The dependence on the concentration parameter $c$ is obtained. It is shown that neglect of this dependence can lead to an overestimation of $\rho_{0}$ by a factor of more than 2 for systems with small $c$. Such errors can strongly influence the estimate of the frequency of formation of double stars in the cores of globular clusters.

\section{INTRODUCTION}

In the modified isothermal model of King of spherical star systems, the spatial density at the center is given by [1]

$$
\rho_{0}=\frac{A \sigma^{2}}{4 \pi G r_{c}^{2}}
$$

where $\sigma^{2}$ is the central one-dimensional velocity dispersion, $G$ is the gravitational constant, and $r_{c}$ is a characteristic scale, called the core radius. In King's opinion, the typical value of $A$ is near 9. However, this is true only for systems with large concentration parameter $c=r_{t} / r_{c}\left[r_{t}\right.$ is the limiting (tidal) radius] that are nearly isothermal. In this case, $r_{c}$ is approximately equal to the distance at which the surface brightness is half the central value.

If Eq. (1) is used to estimate the central densities of star clusters with small $c$, the result can differ appreciably from the true value. At the same time, the central density is one of the most important properties of star systems, and the rate of several dynamical processes in their cores depends on it. As an example, we can mention the process of formation of binary stars, the frequency of which is very sensitive to the estimate of the central density. It would therefore be a good idea to make formula (1) more accurate. The aim of the present note is to obtain values of $A$ with allowance for its dependence on the concentration parameter. An intermediate step in this goal is to determine more accurately the connection between the central value of the potential and the required parameter. This requires numerical integration of the equations of King's model. In fact, in the process the model itself will be improved.

\section{FORMULA FOR ESTIMATING $A$}

The need for a solution to this problem was first pointed out in [2]. Unfortunately, the discussion was based on an incorrect criticism of King's well-known formula for the spatial density of stars in spherical clusters [3]. In particular, it was incorrectly stated that this formula was obtained by an incorrect expression of Abell's equation relating this density [4]

$$
\begin{gathered}
\rho(r)=\rho_{0}(\lambda / z)^{3} \cdot Q(z) / Q(\lambda), \\
\lambda=\left(1+c^{2}\right)^{-1 / 2}, \quad z=\lambda\left(1+r^{2} / r_{c}^{2}\right)^{1 / 2} . \\
Q(x)=\cos ^{-1} x-x\left(1-x^{2}\right)^{1 / 2}
\end{gathered}
$$

to the apparent surface density of the system, which has the form

$$
f\left(r^{\prime}\right)=k \lambda^{2}\left(\frac{1}{z^{\prime}}-1\right)^{2}, \quad z^{\prime}=\lambda\left(1+r^{\prime 2} / r_{c}^{2}\right)^{1 / 2}
$$

In the expressions (2)-(5), $r$ and $r^{\prime}$ are, respectively, the spatial and apparent distance from the center of the system, and $k$ is a constant related to the density at the center by

Byurakan Astrophysical Observatory. Translated from Astrofizika, Vol. 34, No.3, pp. 459-465, May-June, 1991. Original article submitted March 9, 1991; accepted for publication May 21, 1991. 
TABLE 1

\begin{tabular}{l|c|c|c|c}
\hline$W_{0}$ & $c$ & $\lg c$ & $\lg c(\mathrm{~K})$ & $A$ \\
\hline 1 & 3.000 & 0.477 & & 3.844 \\
2 & 3.808 & 0.581 & & 6.334 \\
3 & 5.100 & 0.708 & 0.672 & 7.773 \\
4 & 7.100 & 0.851 & 0.840 & 8.527 \\
5 & 10.78 & 1.031 & 1.029 & 8.856 \\
6 & 18.00 & 1.255 & 1.255 & 8.973 \\
7 & 33.70 & 1.528 & 1.528 & 9.000 \\
8 & 68.10 & 1.833 & 1.833 & 9.000 \\
$9(\mathrm{~K})$ & 131.4 & & 2.119 & 9.000 \\
$10(\mathrm{~K})$ & 223.7 & & 2.350 & 9.000 \\
11 & 353.2 & 2.548 & & 9.000 \\
$12(\mathrm{~K})$ & 548.2 & & 2.739 & 9.000 \\
13 & 855.4 & 2.932 & & 9.000 \\
14 & 1362.5 & 3.134 & & 9.000 \\
$15(\mathrm{~K})$ & 2272 & & 3.356 & 9.000 \\
\hline
\end{tabular}

Note. $(\mathrm{K})$ means that for the given $W_{0}$ the values of the other quantities are taken from King's paper [1]. In the fourth column we give the values of $l o g$ $c$ calculated by King in the same paper under the assumption $A=9$.

$$
\rho_{0}=\frac{k}{\pi r_{c}} Q(\lambda)
$$

The supposedly new formula obtained in [3] for $\rho(r)$, which is equal to zero for $z=1$, is none other than a different definition of (2), as is readily seen. As a result of this, the analytic expression for $A$ deduced in [2] was correct. However, it was used only to establish the boundaries of the region in which the true values of $A$ must lie. An exact solution to the problem was not given.

For this, it is necessary to integrate the system of equations that describe King's model:

$$
\begin{gathered}
\rho / P_{0}=\exp \left(W-W_{0}\right)_{\gamma}\left(\frac{5}{2}, W\right) / \gamma\left(\frac{5}{2}, W_{0}\right), \\
\Delta W=\frac{d^{2} W}{d R^{2}}+\frac{2}{R} \frac{d W}{d R}=-A P / P_{0}, \quad R=r / r_{\varepsilon},
\end{gathered}
$$

where $\gamma$ is the incomplete gamma function

$$
\gamma(\mu, W)=\int_{0}^{W} e^{-y} y^{\mu-1} d y,
$$

$W$ is a dimensionless function equal to $W=\varphi / \sigma^{2}, \varphi$ is the potential, and $W_{0}$ is the value of this function at the center. On $W$ there are imposed the conditions

$$
W^{\prime}(0)=0, \quad W^{\prime}(\mathbf{c})=0 .
$$

The value of $A$ is determined from the condition that in the neighborhood of the center of a system in the isothermal state the theoretical density (7) and the empirical formula (2) agree. If we introduce a certain unit of length $r_{0}$, not necessarily equal to $r_{c}$, then for $A$ we can obtain a more general formula, which in our notation has the form (the corresponding calculations are merely a repetition of those given in [2])

$$
A=\frac{3}{1+\beta\left(W_{0}\right)}\left|\frac{2 \cos ^{-1} \lambda}{Q(\lambda)}+1\right| K^{2}(\lambda), \quad K(\lambda)=\frac{r_{0}}{r_{c}},
$$




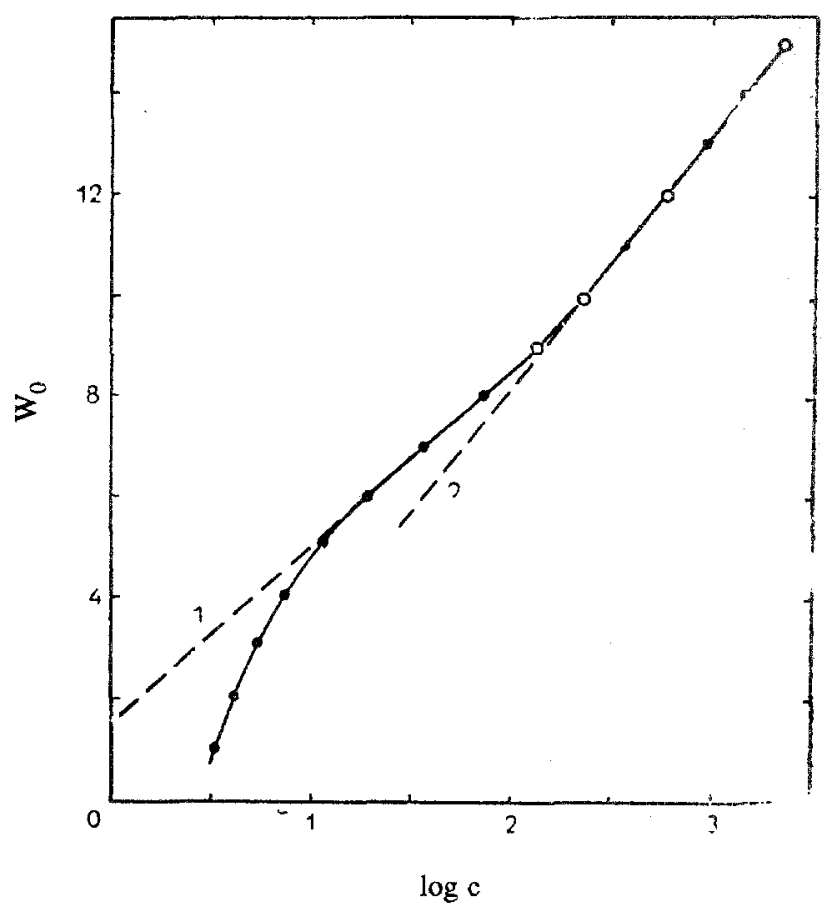

Fig. 1. Plot of $W_{0}$ against $\log c$. The biack circles are our data, the open circles those of King. The straight line (1) corresponds to the first of Eqs. (14).

$$
\beta\left(W_{11}\right)=e^{-W_{0}} W_{0}^{3 / 2} / \gamma\left(\frac{5}{2}, W_{0}\right)
$$

If $r_{0}$ is the distance at which the apparent density is halved compared with its central value (for spatially bounded systems it is not equal to $r_{c}$ ), then, as follows from (5),

$$
K(\lambda)=\sqrt{\left[\frac{1}{\sqrt{2}}(1-\lambda)+\lambda\right]^{-2}-1 .}
$$

For $r_{0}=r_{t}$, we have $K(\lambda)=c$. The case $r_{0}=r_{c}$ leads to the expression for $A$ found in [2]. In the space of the parameters $W_{0}, c$, $A$, the function $A=A\left(W_{0}, c\right)$ describes a surface for which the plane $A=9$ is asymptotic (as $\left.W_{0} \rightarrow \infty, c \rightarrow \infty\right)$.

\section{RESULTS}

Substituting (11) in (8) and solving the system of equations (7) and (8), for each value of $W_{0}\left(W_{0}\right.$ is a free parameter) we can find the unique value of $c$ for which the solution $W$ vanishes at the boundary of the system [see (10)]. After this, $A$ can be calculated. Integration of Eqs. (7) and (8) leads to the values of $c$ and $A$ given in Table 1. The data of the table enable us to represent the relationship between $W_{0}$ and $\log c$ in graphical form (Fig. 1). Determining from observations the concentration parameter, we can approximately calculate $W_{0}$ (and hence $A$ ) without solving the equations of the model. Figure 1 reveals two sections that can be approximated by linear functions:

$$
W_{0}=\left\{\begin{array}{l}
(1.591 \pm 0.079)+(3.508 \pm 0.045) \lg c, 1.25 \leqslant \lg c \leqslant 2.25 \\
(-1.895 \pm 0.157)+(5.066 \pm 0.055) \lg c, \lg c>2.25
\end{array}\right.
$$

The coefficients in (14) were obtained by the least-squares method. The rms error of the approximation for both intervals is 0.057. In these regions of values of $c$, which are typical for globular clusters, it is interesting to compare the approximate estimates $\left[W_{0}(a p)\right]$ obtained by means of [14] with the exact values $W_{0}(\mathrm{ex})$ : 


\begin{tabular}{l|l|l|l|l|l|l|}
\hline $\mathrm{I}_{\mathrm{g}} c$ & 1.25 & 1.50 & 1.75 & 2.00 & 2.25 & 2.50 \\
$W_{\mathrm{n}}(\mathrm{ex})$ & 5.95 & 6.75 & 768 & 8.60 & 9.60 & 10.75 \\
$W_{\mathrm{n}}(\mathrm{ap})$ & 5.977 & 6.854 & 7.731 & 8.608 & 9.485 & 10.77 \\
\hline
\end{tabular}

The obtained numerical results can be used to construct more accurate (both single-component and multicomponent) modified isothermal models, particularly for small $c$.

\section{SOME PHYSICAL CONSEQUENCES}

It follows from the table that for two star clusters with the same $\sigma^{2}$ and $r_{\mathrm{c}}$ the estimates of their central densities in accordance with formula (1), i.e., without allowance for their degree of concentration, can sometimes differ by a factor of more than 2 . The value of $\rho_{0}$ is smaller the smaller the value of the concentration parameter. In the case of an individually considered cluster, formula (1) leads to an overestimation of the central density. If $t_{\mathrm{r}}$ is the relaxation time in the core, then, since $t_{r} \sim \rho_{0}^{-1 / 2}$, this effect alone may result in an overestimation of this time by about 1.5 times (upper limit). In the general case, it is also necessary to take into account the dependence of the central velocity dispersion on the degree of anisotropy in the velocity distribution of the members of the system [5].

For isotropic systems, the neglect of the considered effect can also have a strong influence on the estimate of the frequency of formation in their cores of binary stars. This frequency is very sensitive to the estimate of the number density of single stars. Thus, if binaries are formed in triple encounters [6], the frequency is $\left(d n_{\mathrm{b}} / d t\right) \sim \rho_{0}^{3}$, and, therefore, the overestimation of this quantity can reach an order of magnitude. But if binaries are formed by tidal capture [7], for which $\left(d n_{\mathrm{b}} / d t\right) \sim \rho_{0}^{2}$, then the frequency may be overestimated by about $4-5$ times (upper limit).

\section{CONCLUSIONS}

In this paper, we have numerically integrated the system of equations of the modified isothermal model of King with allowance for the analytic dependence of $A$, which occurs in formula (1), on the finite size of the star system. The results show that the use of formula (1) to estimate the central density of stars in isotropic star clusters under the assumption that $A$ is always equal to 9 leads to a systematic overestimation of $\rho_{0}$, which may exceed the true value of this quantity by two or more times (for clusters with small value of the concentration parameter). This circumstance must be taken into account in estimating the frequency of formation of binary stars in the cores of globular clusters.

\section{REFERENCES}

1. I. King, Astron. J., 71, 64 (1966).

2. A. S. Rastorguev, Astron. Tsirk., 995, 2 (1978).

3. A. S. Rastorguev, Astron. Tsirk., 95, 2 (1978).

4. I. King, Astron. J., 67, 471 (1962).

5. D. Merrit, Astron. J., 95, 436 (1989).

6. D. Heggie, Globular Clusters, Cambridge University Press (1980), p. 281.

7. W. H. Press and S. A. Teukolsky, Astrophys. J., 213, 183 (1977). 